\title{
Attractors synthesis for a Lotka-Volterra like system
}

\author{
Marius-F. Danca ${ }^{1,2}$ \\ ${ }^{1}$ Department of Mathematics and Computer Science, \\ Avram Iancu University, \\ Ilie Macelaru 1A, 400380 Cluj-Napoca, Romania and \\ ${ }^{2}$ Romanian Institute of Science and Technology, \\ Ciresilor 29, 400487 Cluj-Napoca, Romania
}

March 16, 2013

\begin{abstract}
The aim of this paper is to prove numerically, via computer graphic simulations, that the synthesis algorithm provided by Danca et al. in [M.-F. Danca, W. K. S. Tang, G. Chen, A switching scheme for synthesizing attractors of dissipative chaotic systems, Appl. Math. Comput. 201 (2008) 650-667] can be utilized to synthesize any attractor of a dynamical system modeling a two-predator, one prey Lotka-Volterra like system. The algorithm switches in a periodic deterministic or a random way the control parameter inside a set of a chosen values. The obtained attractor is the same with the attractor obtained for parameter value taken as averaged value of the switched control values. This simple and effective algorithm relies on a convex property induced in the set of the attractors corresponding to the chosen switching parameters. The algorithm was tested successfully on systems depending linearly on the control parameter like Lorenz, Chen, Rossler, networks and other systems.
\end{abstract}

keywords: stable attractors, chaotic attractors, Lotka-Volterra system, parameter switching

PACS classification codes: 05.45.Ac, 05.45.Pq, 42.62.Be

\section{Introduction}

In [1] we provided, via numerical approach and computer simulations, that switching the control parameter every finite time intervals in dynamical systems modeled by the following initial value problem which depend linearly on the control parameter

$$
\dot{x}=f_{p}(x), \quad x(0)=x_{0},
$$

where $p \in \mathbb{R}$ and $f_{p}: \mathbb{R}^{n} \longrightarrow \mathbb{R}^{n}$ has the expression

$$
f_{p}(x)=g(x)+p M x,
$$

with $g: \mathbb{R}^{n} \longrightarrow \mathbb{R}^{n}$ a continuous nonlinear function, $M$ a real constant $n \times n$ matrix, $x_{0} \in \mathbb{R}^{n}$, and $t \in I=[0, \infty)$, while some numerical method for ODE is applied to (1), the obtained attractor is identical to one of the system attractors. 
This class of dynamical systems is enough large containing known systems such as Lorenz, Rőssler, Chen, Lotka-Volterra like systems, electronic circuits, systems modeling the terrestrial magnetism, networks, etc.

As it is known, a trajectory (called also in a great majority of standard text books orbit) represents all points in the graph of a single solution to a considered differential equation. Geometrically, it represents the curve along which a solution follows as time changes. In the uniqueness case, to each solution corresponds a unique global attractor (see Appendix). In this paper, for convenience, we shall omit unless necessary the word "global" and simply call attractors the orbits (actually their numerical approximation after neglecting a sufficiently long period of transients [2]) obtained by a numerical method for ODEs with fixed step size $h$ after the transients were neglected. In this purpose, all the numerical experiments were made considering the same attraction basin for the initial conditions.

Despite the fact that there are differences between computation and theory, nonetheless, the numerical integration of (1) via computer graphic simulations can give generally excellent approximations to the orbits within the invariant sets [3]. Also, the orbits that start near a hyperbolic attractor will stay near and they will be shadowed by orbits within the attractor because attractors arise as the limiting behavior of orbits. Therefore, the shadowing property of hyperbolic sets [4] enables us to recover long time approximation properties of numerical orbits.

We shall consider throughout the paper the following assumptions: the existence and uniqueness of solutions on the maximal existence interval $I$ are verified and there exist only hyperbolic equilibria.

Remark 1 It is known that numerical methods for ODEs may correctly reproduce different kinds of limit sets that are present in the differential equations. The required conditions include asking that the behavior in the continuous system be structurally stable. Generaly this condition is not satisfied for the LotkaVolterra systems, which is why the numerical methods used do not correctly reproduce the behavior of that system. Also, the problem with discretization induced by numerical methods, is that it introduces new limit-set behaviors in addition to that already existing in the underlying continuous system. However, the accuracy and the biologically meaningful of our numerical results make it worth the effort and overcome the above possible inconvenience.

The algorithm presented in this paper consists in use a time varying, more preciously periodically or even randomly switching parameter, according to some design rule. It will be demonstrated, empirically by various experiments, that a desired attractor can be duly obtained by the proposed switching scheme.

The organization of the paper is as follows: Section 2 describes shortly the synthesis algorithm, Section 3 presents his application to the case of a Lotka-Volterra three dimensional system and Section 4 summarizes the results of this paper and sketches possible future research directions for this algorithm and system.

\section{Synthesis algorithm}

Notation 2 Let $\mathcal{A}$ be the set of all global attractors depending on parameter $p$, including attractive stable fixed points, limit cycles and chaotic (possibly strange) attractors; $\mathcal{P} \subset \mathbb{R}$ be the set of the corresponding admissible values of $p$ and $\mathcal{P}_{N}=\left\{p_{1}, p_{2}, \ldots, p_{N}\right\} \subset \mathcal{P}$ a finite ordered subset of $\mathcal{P}$ containing $N$ different values of $p$, which determines the set of attractors $\mathcal{A}_{N}=\left\{A_{p_{1}}, A_{p_{2}}, \ldots, A_{p_{N}}\right\} \subset \mathcal{A}$.

We assume that we can access all the values of $\mathcal{P}_{N}=\left\{p_{1}, p_{2}, \ldots, p_{N}\right\}$ for which the system behaves stable and/or chaotic. 
Following the above assumptions, it is naturally to define a bijection between the sets $\mathcal{P}$ and $\mathcal{A}$. Thus, giving any $p \in \mathcal{P}$, a unique global attractor is specified, and vice versa.

Choosing a finite subset $\mathcal{P}_{N}=\left\{p_{1}, p_{2}, \ldots, p_{N}\right\}$ the deterministic synthesis algorithm relies on the following deterministic time switching rule which is applied indefinitely while the numerical method for IVP (1) generates the attractor

$$
\left[\left(m_{1} h\right) p_{\varphi(1)},\left(m_{2} h\right) p_{\varphi(2)}, \ldots,\left(m_{N} h\right) p_{\varphi(N)}\right],
$$

where the "weights" $m_{i}$ are some positive integers and $\varphi$ permutes the subset $\{1,2, \ldots, N\}$. The algorithm acts as follows: in the first time subinterval of length $\Delta t_{1}=m_{1} h, p$ will take the value $p_{\varphi(1)}$, for the next $m_{2}$ integration steps, $p=p_{\varphi(2)}$ (Fig. 1) and so on until the $N$-th time subinterval of length $m_{N} h$ where $p=p_{\varphi(N)}$ after which the algorithm repeats. Thus, the relation (3) is $\left(m_{1}+m_{2}+\ldots+m_{N}\right) h$ periodic. In order to simplify the notation, for a fixed step size $h$, the scheme (3) will be denoted hereafter

$$
\left[m_{1} p_{\varphi(1)}, m_{2} p_{\varphi(2)}, \ldots, m_{N} p_{\varphi(N)}\right] .
$$

For example, by the scheme $\left[1 p_{3}, 3 p_{1}, 2 p_{2}\right]$ one should understand the infinite sequence of $p: 1 p_{3}, 3 p_{1}, 2 p_{2}, 1 p_{3}$, $3 p_{1}, 2 p_{2}, \ldots$ which means that while the considered numerical method integrates $(1), p$ switches in each $m_{i} h$ time subinterval between values of $\mathcal{P}_{3}=\left\{p_{1}, p_{2}, p_{3}\right\}$. The algorithm will generate a synthesized attractor, $A^{*}$, which belongs to $\mathcal{A}$.

To prove that $A^{*} \in \mathcal{A}$ we prove that $A^{*}$ is identical ${ }^{1}$ to the averaged attractor denoted $A_{p^{*}}$ with

$$
p^{*}=\frac{\sum_{k=1}^{N} p_{\varphi(k)} m_{k}}{\sum_{k=1}^{N} m_{k}} .
$$

If we denote $\alpha_{k}=m_{k} / \sum_{k=1}^{N} m_{k}$ it is easy to see that $p^{*}$ is a convex combination $p^{*}=\sum_{k=1}^{N} \alpha_{k} p_{\varphi(k)}$ since $\sum_{k=1}^{N} \alpha_{k}=1$, fact which represents a major property and advantage. Therefore $p^{*} \in\left(p_{1}, \ldots, p_{N}\right)$, whatever the values $p_{i}$ are chosen.

Also, taking into account the bijection between $\mathcal{P}$ and $\mathcal{A}$, we are entitled to consider that the same convex structure is preserved into $\mathcal{A}$. Therefore, for whatever switched values of $p$ in $\mathcal{P}_{N}$, the synthesized attractor $A^{*}$ will belong inside the ordered set $\mathcal{A}_{N}$, endowed with the order induced by the mentioned bijection.

Remark 3 i) The size of the integration step $h$ is an important parameter which may influence the results due the convergence properties of the considered method for ODEs. Obviously, both attractors $A^{*}$ and $A_{p^{*}}$ are simulated with the same fixed step size and same initial conditions.

ii) To relative large values for $m$ or large $N$ may correspond differences between the two attractors $A^{*}$ and $A_{p^{*}}$ [1]. However, in these cases, $A^{*}$ still remains inside of a relative thin neighborhood of $A_{p^{*}}$.

The pseudocode of the synthesis algorithm for chosen $N, T_{\max }, h, m_{1}, \ldots, m_{N}, p_{\varphi(1)}, \ldots p_{\varphi(N)}$ is depicted in Fig. 2

\footnotetext{
${ }^{1}$ By identity will understand a perfect as possible overlap of orbits, histograms and Poincaré sections, taking account on some inherent errors caused by the numerical computation or the screen resolution.
} 


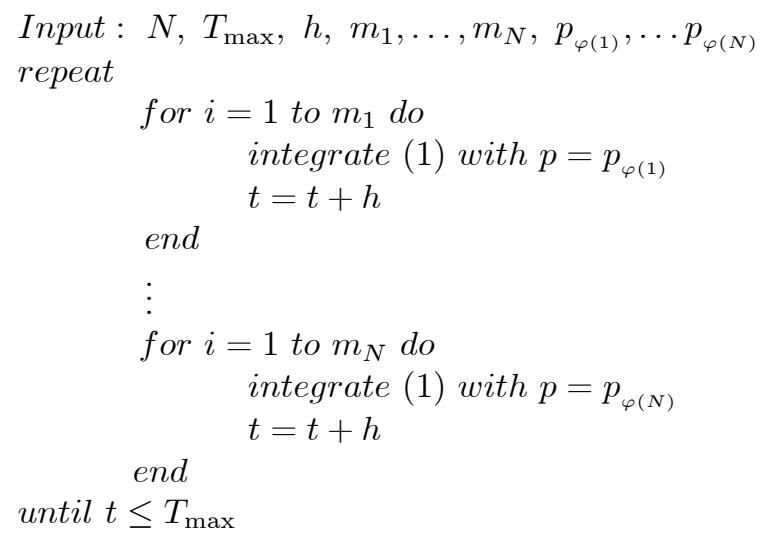

Fig. 2

To support the identity between $A^{*}$ and $A_{p^{*}}$, which verifies the fact that $A^{*}$ belongs to $\mathcal{A}$, histograms, Poincaré sections and phase plots were utilized.

For example, with the scheme $\left[1 p_{1}, 2 p_{2}\right]$ applied to the Lorenz system with $p_{1}=82, p_{2}=98.5$, $p^{*}=\left(1 * p_{1}+2 * p_{2}\right) /(1+2)=93$, the synthesized attractor $A^{*}$ obtained by the synthesis algorithm is similar to $A_{93}$ (see Fig. $3 \mathrm{~b}$ left side where $A^{*}$ and $A_{93}$ are overplotted and Fig. $3 \mathrm{~b}$ right side, where their histograms are overplotted). Remark that even $A_{p_{1}}$ and $A_{p_{2}}$ are chaotic (Fig. 3a), the synthesized attractor $A^{*}$ is periodic (stable limit cycle). However, the scheme $\left[2 p_{1}, 1 p_{2}\right]$ will give $p^{*}=87.5$ (Fig. 3c) for which $A^{*}$ is a chaotic attractor. Because of infinite time required to draw "entirely" chaotic attractors (asymptotic property), there is a relative small difference between the two attractors, $A^{*}$ and $A_{p^{*}}$ (see Poincaré section with the plane $x_{3}=130$ in Fig. 3c).

If the scheme (4) is applied in a random way introduced in [5], the synthesis algorithm generates again an attractor $A^{*}$ which, based on the mentioned above convexity property, will belong inside the set of considered attractors $\mathcal{A}_{N}$ endowed with the order induced by the order of the parameter values in $\mathcal{P}_{N}$. Let us consider again the Lorenz system with $N=2, \quad p_{1}=130$ and $p_{2}=170$ (the attractors $A_{130}$, and $A_{170}$ are plotted in Fig. $4 \mathrm{a}, \mathrm{b}$ ). Using the scheme (4) in some random way (see Fig. 5 where rand means some random procedure with uniform distribution generating the numbers 1 or 2) the synthesized attractor $A^{*}$ will be identical to $A_{p^{*}}$ with $p^{*}=150$. Both attractors are overplotted in Fig. 4c while the overplotted histograms and Poincaré sections (Fig. 4 d,e) underline this identity.

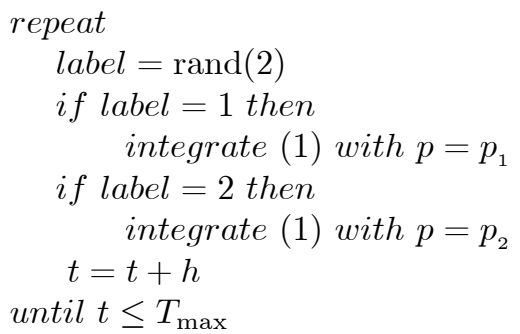

Fig. 5 
Remark 4 i) The synthesis algorithm should not be considered as being a control algorithm since, before the algorithm starts, the system can evolve stable and then the algorithm just change the behavior from a stable attractor to another one. Therefore this algorithm could be viewed as a kind of "general" stabilization because either the system evolves initially stable or chaotic, the algorithm may switch that behavior to another one [6].

ii) The synthesis algorithm can be use to chaotification [1]. Regarding the control/anticontrol characteristics of synthesis algorithm, it should be notified that it can be used when a targeted stable/unstable attractor value of $p$ cannot be set directly.

iii) One of the most important characteristics of the synthesis algorithm is the fact that it may explain how in real systems, belonging to the class modeled by (1), deterministic or random parameter switches may induce chaos or stable behaviors.

Let the sets $\mathcal{P}_{N}$ and $\mathcal{A}_{N}$. Suppose that certain targeted value of $\widehat{p} \notin \mathcal{P}_{N}$ cannot be accessible. Then, using the bifurcation diagram for the considered dynamical system, it is possible to synthesize an attractor corresponding to $\widehat{p}$ using the synthesis algorithm with the values of $\mathcal{P}_{N}$. The only sufficient (and necessary too [1]) condition on $\widehat{p}$ is to belong inside of the real interval $\left(p_{1}, \ldots, p_{N}\right)$ ( $\widehat{p}$ cannot be chosen outside of $\left(p_{1}, \ldots, p_{N}\right)$, because of convexity property). Next, to synthesize the attractor $A_{\widehat{p}}$, we must choose $m_{i}$. Thus, having $p_{1}, \ldots, p_{N}$ fixed, the equation (5) for $p^{*}=\widehat{p}$ with the unknown $m_{i}$ has to be solved. With the obtained values for $m_{i}$, the scheme (4) is next applied. The synthesized attractor $A^{*}$ is identical, as shown above, to $A_{\widehat{p}}$. Thus, using the synthesizes algorithm one can force the system to evolve on the desired stable or unstable orbit corresponding to $\widehat{p}$.

Also, the following situation is possible: $\mathcal{P}_{N}$ is not set a priori. Then, $m_{i}$ and $\mathcal{P}_{N}$ have to be determined such that relation (5) be verified with the only known $\widehat{p}$.

The solutions are not unique in both situations because the elements of $\mathcal{P}_{N}$ belong in a compulsory way to one of the infinite number of $p$-intervals which compose $\mathcal{P}$.

For example, suppose we want to synthesize, with the scheme $\left[m_{1} p_{2}, m_{2} p_{1}\right]$, a stable orbit for the Lorenz system, corresponding to $\widehat{p}=150$ and starting from $\mathcal{P}_{N}=\{130,170\}$. Then, one of the possible solutions to (5), is $m_{1}=2$ and $m_{2}=1$.

\section{Synthesis of Lotka-Volterra attractors}

The original system discovered by both Volterra [7] and Lotka [8] independently, consisted of two entities. Vito Volterra developed these equations in order to model a situation where one type of fish is the prey for another type of fish [9]. This model was generalized by Smale [10] for dimensions $n \geq 3$.

Nowadays, there are several three-dimensional competitive Lotka-Volterra systems which are proposed to provide a simplified model of two-species predator and one prey population dynamics ${ }^{2}$. The equations exhibit complex oscillatory behavior (mimicking the boom-and-bust cycles that species in a predator/prey relationship are presumed to undergo, though these cycles seem not to be empirically observable, at least in the form they appear in the dynamics of Lotka-Volterra). In a 1988 paper [11] Samardzija and Greller propose a biologically realistic two-predator, one prey generalization model of the Lotka-Volterra equations on which we focus in this paper (we refer also [12])

\footnotetext{
${ }^{2}$ This system may be interpreted as a chemical, biochemical, or ecological model.
} 


$$
\begin{aligned}
& \dot{x}_{1}=x_{1}-x_{1} x_{2}+b x_{1}^{2}-a x_{3} x_{1}^{2}, \\
& \dot{x}_{2}=-x_{2}+x_{1} x_{2}, \\
& \dot{x}_{3}=-p x_{3}+a x_{3} x_{1}^{2},
\end{aligned}
$$

where $a, b, p$ are positive parameters. In the real world, these values would certainly not remain constant. The time of day and the current season affect these values. For example certain animals only mate during certain seasons, some animals hibernate or migrate when the seasons change, some animals hunt by day, and others are nocturnal and so on. For these reasons all parameters should be functions of $t$ rather than constants, but the complexity of (6) is daunting. The (6) model remains tractable by letting $a, b$ values be constant rather than functions this particularization being enough realistic by biologic point of view. In this paper, we consider $p$ as being the control parameter, while all the other parameters are kept constant as presented in [11]: $a=2.9851$ and $b=2$.

The stability of the fixed points are established in [11] where it is shown that in a wide range of parameters the behavior is chaotic.

The most intriguing attractor of this system is, with no doubt, the fractal torus (characterization introduced in [11]). This particular attractor exhibited by system (6) for certain values of $a, b$ and $p$ is interesting because has a structure different from attractors such as the Rössler, Lorenz or Chen attractors (see e.g. Fig. 9). This typical attractor consists of a slow and a fast manifold, shaped like a sphere, the slow dynamics being confined to a very skinny tube on the inside of the attractor, while the fast oscillatory dynamics being on the outside surface of attractor. Also, all numerical experiments reveal the fact that the initial conditions play an important role leading to several different fast manifolds, the slow manifolds being all tangled up along one line. The shape of the fractal torus suggests a quasiperiodic motion.

Even the higher dimensional Lotka-Volterra models, compared to the usual two dimensional LotkaVolterra Equations, are more complicated as one might think and have a bad reputation among biologists as being oversimplified and simplistic models, the proposed variant allows positive feedback among the species.

While the dynamics of three-dimensional Lotka-Volterra systems are well known and studied extensively in several works, in this paper we focus on the behavior of (6) when the control parameter is switched, via the synthesis algorithm, in a deterministic and random manner.

The standard Runge-Kutta method with fixed step size was utilized. To sustain the accuracy of the results, beside phase portraits, histograms and Poincaré sections were utilized. The integration step size was $h=10^{-4} \div 10^{-2}$.

Remark 5 Because of the pronounced asymptotic character of these attractors, however long time was fixed to integrate the system, the results have never been obtained with enough accuracy. However, acceptable results were found for $T_{\max }=10000$.

To facilitate the attractors synthesis, the bifurcation diagram was determined (Fig. 6). In the same figure, all the attractors utilized to synthesis are plotted in the bottom, while the synthesized attractors in the top. The results are presented in Table 1. In the first three considered cases, $N=2$ (Fig. 7-9) while in the forth case (Fig. 10) the algorithm is applied for $N=4$, to underline the robustness of the algorithm. In Fig. 11 is obtained the same attractor $A^{*}$ like in Fig.10 with the random way presented in Fig. 5 , for $p_{1}=3$ and $p_{2}=4$. 


\begin{tabular}{||c|c|c|c|c|c|c||}
\hline \hline Scheme & $p_{1}$ & $p_{2}$ & $p_{3}$ & $p_{4}$ & $p^{*}$ & Remarks \\
\hline$\left[1 p_{1}, 1 p_{2}\right]$ & 2.9 & 3.7 & - & - & 3.3 & $A_{p_{1}}, A_{p_{2}}$ stable, $A^{*}$ chaotic (Fig. 7) \\
\hline$\left[1 p_{1}, 1 p_{2}\right]$ & 2.8 & 3.2 & - & - & 3 & $A_{p_{1}}$ stable, $A_{p_{2}}$ chaotic, $A^{*}$ Fractal torus (Fig. 8) \\
\hline$\left[1 p_{1}, 1 p_{2}\right]$ & 3 & 5 & - & - & 4 & $A_{p_{1}}$ Fractal torus, $A_{p_{2}}$ stable, $A^{*}$ stable (Fig. 9) \\
\hline$\left[1 p_{1}, 1 p_{2}, 2 p_{3}, 3 p_{4}\right]$ & 1.3 & 3 & 4 & 5 & 3.9 & $A_{p_{1,3,4}}$ stable, $A_{p_{2}}$ Fractal torus $A^{*}$ stable (Fig. 10) \\
\hline random & 3 & 4 & - & - & 3.9 & $A_{p_{1}}$ Fractal torus, $A_{p_{2}}$ stable, $A^{*}$ stable (Fig. 11) \\
\hline \hline
\end{tabular}

Table 1

\section{Conclusions and further directions}

In this study, we verified numerically that any attractor of the Lotka-Volterra system (6) can be synthesized by the synthesis algorithm.

This algorithm may be used to explain what is happens in a system like Lotka-Volterra if, accidentally or intentionally, the control parameter switches in time.

Thus, if we switch periodically the control parameter following a determinist rule, the orbit can be forced to reach a desired stable or unstable attractor. The control parameter is switched inside a finite subset $\mathcal{P}_{N}$ of the set of all admissible values $\mathcal{P}$, every finite time subintervals. The synthesized stable attractor is identical to an attractor, belonging to the set of all attractors $\mathcal{A}$, which corresponds to the averaged value of the switched values of $\mathcal{P}_{N}$.

Mathematical studies on this algorithm remain a task for future works such as the convergency of the synthesized attractor $A^{*}$ to the averaged attractor $A_{p^{*}}$, the study of the step size influence, and so on.

\section{Appendix}

Definition 6 A global attractor of a dynamical system is a compact set composing of all bounded global trajectories of system (1) (see e.g. [13]).

Definition 7 A local attractor for the dynamical (1) is a compact set, invariant under $f_{p}$, which attracts its neighboring trajectories (see e.g.[14]).

A global attractor can be considered, roughly speaking, as a region of a dynamical system's state space that the system can enter but not leave, and which contains no smaller such region. From the definition, a global attractor contains all the dynamics evolving from all possible initial conditions. In other words, it contains all solutions, including stationary solutions, periodic solutions, as well as chaotic attractors, relevant to the asymptotic behaviors of the system.

The terminology local attractor is sometimes used for attractors which are not global attractors. A global attractor may contain several local attractors. A global attractor is hence considered as being composed of the set of all local attractors, where each local attractor only attracts trajectories from a subset of initial conditions, specified by its basin of attraction. Therefore, for a fixed parameter $p$, different local attractors may be obtained depending on the choice of the initial condition, in contrast to the uniqueness of the case of a single global attractor. For example, if one considers the Lorenz system with the control parameter $r=2.5$, there are three local attractors: the origin (saddle) and two symmetrical fixed points (sinks) $X( \pm 2, \mp 2,1.5)$. A unique local attractor may also be the global attractor. For the 
same example of Lorenz system, when $r=28$, there exists only a single local attractor, which is a global attractor too (known as the Lorenz strange attractor).

More details and background on attractor notion can be found in [15].

\section{References}

[1] M.-F. Danca, W.K.S. Tang, G. Chen, A switching scheme for synthesizing attractors of dissipative chaotic systems, Appl. Math. Comput. 201 (2008) 650-667.

[2] C. Foias, M.S. Jolly, On the numerical algebraic approximation of global attractors, Nonlinearity 8 (1995) 295-319.

[3] A.M. Stuart, A. R. Humphries, Dynamical systems and numerical analysis, Cambridge University Press, Cambridge, 1996.

[4] B. Coombes, H. Kocak, K. Palmer, Rigorous computational shadowing of orbits of ordinary differential equations, Numer. Math. 69 (1995) 401-421.

[5] M.-F. Danca, Random parameter-switching synthesis of a class of hyperbolic attractors, Chaos 18 (2008) 033111.

[6] M.-F. Danca, Finding stable attractors of a class of dissipative dynamical systems by numerical parameter switching, Dynamical Systems (2009) DOI 10.1080/14689360903401278.

[7] V. Volterra, Leçons sur la théorie mathématiques de la lutte pour la vie, Gauthier-Villars, Paris, 1931.

[8] A.J. Lotka, Undamped oscillations derived from the law of mass action, J. Am. Chem. Soc. 42 (1920) 1595-1599.

[9] W.E. Boyce, R.C. DiPrima, Elementary differential equations and boundary value problems, 8th ed., John Wiley \& Sons, Inc., New York, 2005.

[10] S. Smale, On the differential equations of species in competition, J. Math. Biol. 3 (1976) 5-7.

[11] N. Samardzija, L.D. Greller, Explosive route to chaos through a fractal torus in a generalized LotkaVolterra model, Bulletin of Mathematical Biology 50 (1988) 465-491.

[12] R.W. May, W.J. Leonard, Nonlinear aspects of competition between three species, SlAM J. Appl. Math. 29 (1975) 243-253.

[13] L. Kapitanski, I. Rodnianski, Shape and Morse theory of attractors, Commun. Pur. Appl. Math. 53 (2000) 218-242.

[14] W.M. Hirsch, S. Smale, R.L. Devaney, Differential equations, dynamical systems and an Introduction to chaos, second ed., Elsevier Academic Press, New York, 2004.

[15] J. Milnor, On the concept of attractor, Communications in Mathematical Physics 99 (1985) 177-195. 


\section{Figure Captions}

Fig. 1 Synthesis algorithm (sketch).

Fig. 2 Pseudocode of synthesis algorithm

Fig. 3 Synthesis algorithm applied for the Lorenz system with the scheme $\left[1 p_{1}, 2 p_{2}\right]$; a) Attractors $A_{p_{1}}$ and $A_{p_{2}}$ for $p_{1}=82, p_{2}=98.5 ;$ b) The synthesized and averaged attractors $A^{*}$ and $A_{p^{*}}\left(p^{*}=\right.$ 93) overplotted (left) and overplotted histograms (right); c) Overplotted attractors $A^{*}$ and $A_{p^{*}}\left(p^{*}=\right.$ 87.5) obtained with the scheme $\left[2 p_{1}, 1 p_{2}\right]$ (left) and the overplotted Poincaré section with the plane $x_{3}=$ 130 (right).

Fig. 4 The synthesized and averaged attractors $A^{*}$ and $A_{p^{*}}$ obtained with the random way described in Fig. 5 with $N=2, \mathcal{P}_{2}=\{130,170\}$; a, b) The attractors $A_{130}$ and $A_{170} ;$ c) Attractors $A^{*}$ and $A_{p^{*}}$ overplotted with $p^{*}=150 ;$ d) Overplotted histograms; e) Overplotted Poincaré sections with the plane $x_{3}=130$.

Fig. 5 Pseudocode for a random variant of the synthesis algorithm .

Fig. 6 Bifurcation diagram for the Lotka-Volterra system with the attractors utilized for synthesis.

Fig. $7 \mathrm{~A}$ chaotic attractor $A^{*}$ synthesized with scheme $\left[1 p_{1}, 1 p_{2}\right]$, for $p_{1}=2.9, p_{2}=3.7$ and $p^{*}=3.3$; a,b) The stable periodic attractors $A_{p_{1}}$ and $A_{p_{2}}$; c) $A^{*}$ and $A_{p^{*}}$ overplotted; d) Overplotted histograms.

Fig. 8 The Fractal torus synthesized with the scheme $\left[1 p_{1}, 1 p_{2}\right]$ for $p_{1}=2.8, p_{2}=3.2$ and $p^{*}=3$; a,b) The stable fixed point attractor $A_{p 1}$ and the chaotic attractor $A_{p_{2}}$; b) The fractal torus $A^{*}$ and $A_{p^{*}}$ overplotted; d) Histograms overplotted.

Fig. 9 Stable attractor synthesized with the scheme $\left[1 p_{1}, 1 p_{2}\right]$ for $p_{1}=3, p_{2}=5$ and $p^{*}=4$; a,b) The fractal torus and stable limit cycle $A_{p 1}$ and $A_{p_{2}}$; c) $A^{*}$ and $A_{p^{*}}$ overplotted;d) Overplotted histograms.

Fig. 10 Stable attractor obtained with the scheme $\left[1 p_{1}, 1 p_{2}, 2 p_{3}, 3 p_{4}\right]$ for $p_{1}=1.3, p_{2}=3, p_{3}=4$ and $p_{4}=5$.Here $p^{*}=3.9$; a-d) Attractors $A_{1-4}$; e) $A^{*}$ and $A_{p^{*}}$ overplotted; f) Overplotted histograms.

Fig. 11 The same stable attractor as in Fig. 10 obtained in the random way presented in Fig. 5.

\section{Table Caption}

Table 1 The Lotka-Volterra attractors synthesized with both deterministic and random algorithms. 